\title{
Rol de los determinantes sociales y económicos en la infección y en la mortalidad por SARS
} Cov2

\section{Role of social and economic determinants in infection and mortality from SARS Cov2}

Al terminar el 2020, la COVID-19 causada por el SARS Cov2 ha generado más de 78 millones de casos, más de 1,7 millones de muertes en el mundo, y probablemente muchos más ya que la curva a nivel global de casos está ascendiendo a casi el doble del pico de la primera ola global en abril del 2020 (1). Aunque esta pandemia ha afectado a países de altos, medianos y de bajos ingresos, existe suficiente evidencia para demostrar que los determinantes sociales y económicos como pobreza, acceso oportuno a servicios de salud, condiciones de vivienda y otros factores ambientales, tienen un impacto en la probabilidad de infectarse y morir por el SARS Cov2.

Considerando que la letalidad por COVID-19 es mayor a medida que la edad aumenta, la estructura etaria de la población tiene también un impacto en la mortalidad. Es por esto que países con bajos niveles de pobreza pero alta proporción de la población en los grupos de mayor edad, como Europa, tengan alta mortalidad, y países con altos niveles de pobreza pero una menor población en los grupos de mayor edad, como algunos de África, tengan bajos niveles de mortalidad. En este número Cieza J. y Uriol C. (2), comparan las variables sociales, demográficas y económicas relacionadas a salud de 60 países con el número de casos y con la mortalidad y la letalidad por COVID-19 en los primeros meses del 2020. Esta variabilidad en la mortalidad de los países y los determinantes sociales y económicos ha sido luego estudiada hasta junio 2020 (3) con hallazgos similares.

Estudiar las tasas de mortalidad y letalidad de una enfermedad es indispensable para dirigir las intervenciones para reducir el riesgo en quiénes tienen mayor probabilidad de morir. El impacto del SARS Cov2 puede medirse por la mortalidad, es decir las muertes que causa en la población, así como por la letalidad, es decir las muertes que causa entre los casos. La letalidad es una medida de la severidad de una enfermedad, mientras que la mortalidad depende de la incidencia de la enfermedad, es decir cuántos casos hay, y de la frecuencia de los factores asociados a mayor letalidad en la población. Como una proporción importante de las infecciones por SARS Cov2 pueden ser asintomáticas, los casos que se diagnostican son solo una fracción de todas las infecciones que suceden, incluso en los países con altas coberturas de tamizaje y diagnóstico. Los países con altas coberturas de tamizaje y diagnóstico, tendrán menor tasas de letalidad por casos que los países que diagnostican una menor proporción de casos leves. Asimismo, al comparar la mortalidad y letalidad por COVID-19, es importante considerar las definiciones usadas en los países para clasificar muertes por COVID-19, la cobertura y calidad de los sistemas de vigilancia y de registros vitales, las definiciones de casos de COVID-19 y el número de pruebas hechas según la población.

Para entender el rol de los determinantes sociales en la infección y en la mortalidad por SARS Cov2 es importante recordar las rutas de transmisión del virus. El SARS Cov2 se transmite de persona a persona a través de secreciones respiratorias, aerosoles, y en mucho menor magnitud, por fómites. Estas formas de transmisión guían las formas de prevención. Para reducir el riesgo de infección por SARS Cov2 debemos reducir los contactos con otras personas, tanto en número como en tipo de contacto (cercano, prolongado, en lugares cerrados, sin mascarilla), usar mascarilla,

Instituto de Medicina Tropical Alexander von Humboldt. Universidad Peruana Cayetano Heredia. Lima, Perú.

Profesor auxiliar.

Magister en Salud Pública, orientación en control de enfermedades tropicales.

Doctor en Ciencias de la Salud 
mantener distancia física de otras personas y lavarse las manos con frecuencia para evitar transportar el virus de las manos a la cara y vías respiratorias altas. Luego, la capacidad del SARS Cov2 de infectar por lo menos dos días antes que la persona inicie síntomas y la alta frecuencia de infecciones asintomáticas o con síntomas muy leves, requiere que las personas se aíslen si tienen síntomas o si han estado en contacto con una persona con síntomas. Para que sea efectivo, este aislamiento debe ser de inmediato y por más de una semana.

Es así que los determinantes sociales afectan el riesgo de infección por SARS Cov2 porque las acciones necesarias para reducir la exposición al virus dependen de la posibilidad de poder quedarse en casa, así como las condiciones de la casa para poder separar a personas de alto riesgo de enfermedad severa por COVID-19 de las personas que pueden traer la infección a la casa. El tener un trabajo que se puede realizar en casa usando una computadora e internet, o un trabajo presencial que brinda las medidas para reducir el riesgo incluyendo aislarse de inmediato y en cualquier momento si un trabajador tiene síntomas o está en contacto con alguien que los tiene, el poder transportarse en una movilidad privada en lugar de compartir el transporte público y una vivienda con electricidad, agua y desagüe y con condiciones mínimas necesarias para el número de personas que viven en ella, tienen un impacto directo en el riesgo de infectarse. Es así como las personas que no tienen un trabajo formal o tienen un trabajo formal con condiciones precarias, tienen menos posibilidad de llevar a cabo las medidas de prevención básicas en esta pandemia. En Estados Unidos, los agricultores, que son en su mayoría migrantes y de minorías raciales y étnicas, tuvieron más riesgo de infectarse y morir por COVID-19 (4). En Inglaterra, el índice de privación múltiple, una medida que incluye ingresos, estado laboral, educación y habilidades, salud, crimen, vivienda, servicios y ambiente, ha sido, después de la edad y las comorbilidades, es el factor más fuertemente asociado con la mortalidad por COVID-19 (5).

El acceso a la salud incluye la existencia de servicios de salud que tengan una infraestructura y calidad suficientes para tener la capacidad de resolver los problemas de salud de la comunidad y en la casa de manera oportuna, segura y aceptable. Asimismo, además de cubrir los costos de la prestación en salud por seguros públicos o privados, las personas tienen que poder cubrir los costos de oportunidad por acudir a los servicios de salud (ingresos no recibidos durante el tiempo en el servicio de salud, cuidado de los niños, transporte, entre otros). En Estados Unidos, los costos de bolsillo influirían en la búsqueda de atención en salud en caso de desarrollar síntomas de COVID-19, en dos tercios de mil personas encuestadas (6).

Es importante resaltar que dado que la pandemia está en curso, aún no podemos establecer con certeza cuáles son los determinantes más importantes en la transmisión y mortalidad por COVID-19. Países con determinantes sociales y económicos muy favorables y cuyos gobiernos e instituciones cumplieron con eficiencia las medidas de prevención y control, como lo es Alemania, tienen en este momento una transmisión descontrolada. Otros con similares características, han logrado con preparación y gran esfuerzo, mantener la transmisión aún bajo control como son Corea del Sur, Japón y Taiwán.

Los avances tecnológicos y científicos han permitido un hecho histórico como tener una vacuna muy efectiva y segura en menos de un año. El acceso oportuno a esta vacuna estará también afectado por los determinantes sociales y económicos. Es así que mientras que existan determinantes sociales que afecten tan adversamente la salud individual y colectiva, una gran parte del mundo será inmune a la tecnología, a la ciencia y a las buenas políticas de salud.

\section{Larissa Otero $^{1, \mathbf{a}, \mathbf{b}, \mathbf{c}}$}

\section{REFERENCIAS BIBLIOGRÁFICAS}

1. Roser M, Ritchie H, Ortiz-Ospina E, Hasell J. "Coronavirus Pandemic (COVID-19)". Published online at OurWorldInData.org. (Citado el 20 de diciembre de 2020) Disponible en: https:// ourworldindata.org/coronavirus

2. Cieza J, Uriol C. Letalidad y la mortalidad de Covid 19 en 60 países afectados y su impacto en los aspectos demográficos, económicos y de salud. Rev Med Hered. 2020 oct-dic; 31:214-221.

3. Sorci G, Faivre B, Morand S. Explaining amongcountry variation in COVID-19 case fatality rate. Sci Rep. 2020 Nov 3; 10(1):18909. doi: 10.1038/s41598020-75848-2.

4. Fielding-Miller RK, Sundaram ME, Brouwer K. Social determinants of COVID-19 mortality at the county level. PLoS ONE. 2020; 15(10): e0240151. 
https://doi.org/10.1371/journal.pone.0240151

5. Williamson EJ, Walker AJ, Bhaskaran K, Bacon S, Bates C, Morton CE, Curtis HJ, Mehrkar A, Evans D, Inglesby P, Cockburn J, McDonald HI, MacKenna B, Tomlinson L, Douglas IJ, Rentsch CT, Mathur R, WongAYS, GrieveR, HarrisonD, Forbes H, SchultzeA, Croker R, Parry J, Hester F, Harper S, Perera R, Evans SJW, Smeeth L, Goldacre B. Factors associated with COVID-19- related death using OpenSAFELY. Nature. 2020 Aug; 584(7821):430-436. doi: 10.1038/s41586-020-2521-4.

6. The Commonwealth Fund. What are Americans' views on the coronavirus pandemic? NBC News/ Commonwealth Fund health care poll. 2020. Disponible en: commonwealthfund.org/ publications/surveys/2020/mar/what-are-americansviews-coronavirus-pandemic 